\title{
Quand la création habite l'environnement: Le Toutou de La Vérendrye
}

\section{Maia Morel}

\section{(2) OpenEdition \\ Journals}

Édition électronique

URL : http://journals.openedition.org/ere/2510

DOI : 10.4000/ere.2510

ISSN : 2561-2271

Éditeur

Centr'ERE

Référence électronique

Maia Morel, «Quand la création habite l'environnement : Le Toutou de La Vérendrye », Éducation relative à l'environnement [En ligne], Volume 14 - 1 | 2017, mis en ligne le 15 septembre 2018, consulté le 21 février 2020. URL : http://journals.openedition.org/ere/2510 ; DOI : 10.4000/ere.2510 


\title{
Quand la création habite l'environnement : Le Toutou de La Vérendrye
}

\author{
Maia Morel
}

1 Si les œuvres d'art ont pu sembler à une certaine époque concentrées dans des lieux spécifiques (musées et galeries en particulier), il y a longtemps que les nouvelles pratiques artistiques, comme le Street Art que des créateurs tels que Banksy et Invader ont rendu célèbre ou le Land Art que l'on associe souvent à Andy Goldsworthy, leur ont permis d'investir largement les rues et l'espace naturel. Les voies de communication semblent à cet égard représenter un lieu privilégié par le fait qu'elles sont fréquentées par de nombreux voyageurs d'horizons très divers, et qu'elles ont de tout temps fait l'objet d'une attention spéciale de la part des autorités (qu'on songe par exemple aux arcs de triomphe ou aux calvaires). Les sociétés commerciales ont été parmi les premières à s'y intéresser et tous ceux qui ont traversé l'Espagne se rappellent les immenses taureaux Osborne qui bordent certaines routes. Au nombre de 91, ces silhouettes servent d'abord de publicité pour une marque d'alcool, mais elles ont également acquis, depuis une vingtaine d'années, un statut de bien culturel et de symbole politique. En France, l'art routier et autoroutier a même reçu à une certaine époque un appui officiel par application de la loi dite du $1 \%$ artistique qui impose aux sociétés constructrices d'ouvrages publics de consacrer $1 \%$ de leur budget $-1 \%$ pour les autoroutes (République française, 1980, art. 4) - à l'achat d'œuvres d'art. Avec ou sans soutien institutionnel, les lieux de passage ont toujours attiré artistes et créateurs, professionnels ou non.

2 Nous nous attarderons ici sur une installation «improvisée " née dans une réserve faunique au bord d'un axe routier du Québec. Elle nous servira de base à une réflexion sur l'articulation qui peut se créer entre l'art spontané (produit par des non-artistes) et l'environnement. Nous analyserons pour cela les caractéristiques principales de cette installation, les liens qu'elle a établis avec le cadre naturel dans lequel elle s'inscrit, 
avant de nous pencher sur les opportunités qu'elle peut offrir à l'éducation relative à l'environnement (ERE).

\section{L'art routier au Québec}

3 En plus d'un public abondant et relativement accessible, l'accotement routier et tout ce qui appartient à l'emprise de la route ou à son environnement immédiat présentent un caractère de disponibilité qui a tout pour provoquer le créateur. Au Québec, deux productions récentes nous en fournissent un bon exemple :

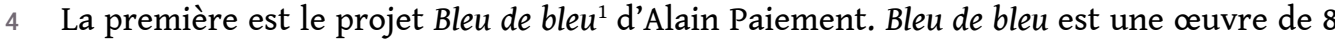
$\mathrm{km}$ de longueur réalisée le long de l'autoroute qui relie l'aéroport international de Montréal au centre-ville ; l'ouvrage est financé par la Banque Nationale et plusieurs autres mécènes. Utilisant principalement le mur antibruit qui borde la chaussée des deux côtés, l'œuvre vise à mettre de l'avant une image de la métropole comme lieu créatif dans le cadre des célébrations de son $375^{\circ}$ anniversaire.

5 Le second exemple est celui de Truck stop ${ }^{2}$, un événement dont le but était de présenter des réalisations artistiques sur le trajet de l'autoroute 20, qui relie Montréal à Québec, du 17 juin au 19 août 2017. Cet ensemble, selon le site Truck stop, « regroup[ait] le travail de 15 artistes des régions de Montréal et de Québec aux pratiques variées. Sculptures, installations, performances, photographies, œuvres sonores et lumineuses [étaient] déployées tout le long de l'autoroute 20 dans les deux directions, intégrées à certaines haltes routières, à une grange, un silo, chez un antiquaire, etc. ».

6 Nous sommes ici sur le territoire de ce que Gilles Clément, ingénieur et paysagiste porteur d'une réflexion qui vise à rendre la nature plus libre et l'homme plus responsable, a appelé le tiers-paysage (2016) :

7 Le Tiers-Paysage - fragment indécidé du Jardin Planétaire - désigne la somme des espaces où l'homme abandonne l'évolution du paysage à la seule nature. Il concerne les délaissés urbains ou ruraux, les espaces de transition, les friches, marais, landes, tourbières, mais aussi les bords de route, rives, talus de voies ferrées, etc. À l'ensemble des délaissés viennent s'ajouter les territoires en réserve. Réserves de fait: lieux inaccessibles, sommets de montagne, lieux incultes, déserts ; réserves institutionnelles : parcs nationaux, parcs régionaux, « réserves naturelles » (Clément, s. d., paragr. 1).

La question que l'on peut se poser est de savoir si le tiers-paysage, lieu d'une nature délaissée, mais libre, ne serait pas aussi celui d'une humanité libre de créer.

Cette question m'a conduite à poser un regard neuf sur l'un de mes «trésors de voyage » : des photos prises le long de la route qui mène en Abitibi (région du nord du Québec), qui m'ont amenée à formuler quelques réflexions sur le phénomène de l'art routier et plus particulièrement sur une œuvre singulière que j'ai appelée : Le Toutou. Nous nous interrogerons ici sur les caractéristiques de cette installation spontanée et unique, née au milieu d'un domaine réservé et qui, contrairement aux deux œuvres mentionnées ci-dessus, n'a pas d'auteur ni de commanditaire connu, pas de but proclamé et pas de budget. Elle n'a pas non plus de valeur commémorative : d'après mes informations, aucun accident n'est répertorié à cet endroit. Après en avoir relevé quelques traits principaux, nous envisagerons plus largement les réserves naturelles comme un autre terrain de l'art, en ce sens qu'elles constituent un lieu atypique propice à la création tout comme à l'ERE. 


\section{Le Toutou de La Vérendrye}

\section{kilomètres 326 et 327 de la route 117 qui relie Montréal à la région de l'Abitibi-} Témiscamingue. Il est donc situé en pleine réserve faunique La Vérendrye ${ }^{4}$, dans un lieu isolé à 50 kilomètres du point d'entrée et à 134 kilomètres du point de sortie. Il se situe à une quinzaine de mètres de la chaussée, hors de l'emprise technique de celle-ci, sur un petit ressaut rocheux qui le rend bien visible sauf en saison hivernale où il est recouvert par la neige.

11 Quelques photographies du Toutou ${ }^{5}$ que j'ai prises entre octobre 2015 et octobre 2017 (ci-dessous) illustrent son évolution au cours du temps : initialement composée d'un gros toutou assis sur un fauteuil, auquel se sont joints par la suite d'autres peluches et quelques objets ou du mobilier (table, lampe, chaise longue, écran d'ordinateur), l'installation représente aujourd'hui toute une mise en scène d'un véritable «monde peluchard».

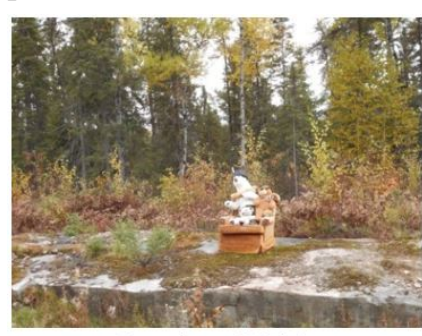

Octobre 2015

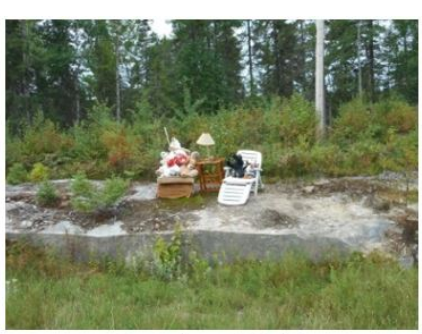

Octobre 2016

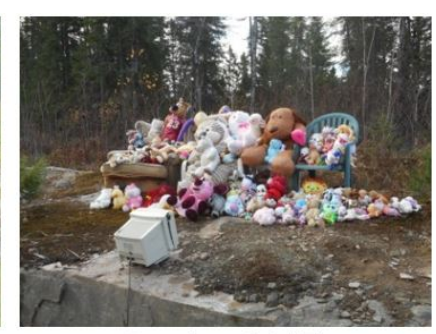

Octobre 2017

12 C'est pour moi une création touchante, qui éveille à la fois des émotions et de tendres souvenirs d'enfance, mais aussi une œuvre qui, sous un regard plus rigoureux et rationnel, rappelle indéniablement plusieurs caractéristiques de l'art contemporain.

\section{L'anonymat}

13 Le Toutou de La Vérendrye fait partie de ce que l'on appelle en art les productions esthétiques clandestines. En effet, afin de savoir qui avait eu l'initiative de cette réalisation et si une personne ou un groupe en assurait la gestion, j'ai interrogé l'administration du parc:

14 Malheureusement nous n'avons aucune idée de la personne qui a eu l'initiative de ce «rassemblement» de peluches. Il y a trois ans environ quelqu'un avait laissé un fauteuil à cet endroit. Par la suite, un chien en peluche a été laissé sur le fauteuil et avec les années d'autres peluches ont été ajoutées [...]. (Courriel du $1^{\text {er }}$ décembre 2017)

15 À cet égard, on pourrait rapprocher Le Toutou de l'art de rue qui, présent dans l'environnement urbain sous diverses formes (graffitis, pochoirs, tricots, etc.), est lui aussi fait des diverses traces laissées par des auteurs souvent inconnus dans le but de contester, d'exprimer, d'intriguer ou de provoquer. Quoi qu'il en soit, Le Toutou-art-deroute touche tout autant notre imaginaire par cet anonymat, dont certains artistes contemporains se sont fait une identité (Bertini, 2015), que par la symbolique qui s'attache à la peluche, proche du duvet ou de la fourrure et riche d'interprétations psychanalytiques. 


\section{L'accumulation}

16 «[...] un chien en peluche a été laissé sur le fauteuil et avec les années d'autres peluches ont été ajoutées [...] », lit-on dans le courriel de l'administration du parc. Dès lors, il n'est pas difficile de deviner le processus : des automobilistes touchés par Le Toutou, ou d'autres voyageurs, enfants en particulier, s'approprient petit à petit l'idée de recueillir à l'occasion une vieille peluche et de l'intégrer dans l'œuvre. Ainsi est lancée la grande collection des toutous : récupérer, rassembler, amasser, agglomérer, etc. Il s'agit là de gestes chers à l'artiste contemporain, dont les racines se trouvent dans l'œuvre de Duchamp, qui au début du XXe siècle, ouvrait le champ artistique au foisonnement des objets du quotidien.

\section{La prolifération}

17 Si à ce jour, Le Toutou incarne l'exubérance, c'est parce qu'on assiste à un mécanisme d'élaboration continu, à la fois spontané et téméraire, vers lequel ont convergé les automobilistes de La Vérendrye, et qui permet d'enrichir et d'agrandir l'œuvre. De manière imperceptible, elle prend constamment de plus grandes proportions. Cette dynamique convoque l'idée de série, de multiplication, de recouvrement, d'invasion, de contamination, d'envahissement, d'obsession, en rappelant des pratiques artistiques contemporaines. À l'instar d'Arman ou Boltanski, qui ont fait de l'empilement et de la propagation leur signe distinctif, les artisans anonymes du Toutou semblent avoir été séduits par ce type de procédé.

\section{L'interactivité}

Fruit d'un travail collectif réalisé par une entité non définie et non constante, Le Toutou n'est pas une création statique. Il ne s'agit pas d'une œuvre finie et exposée en tant que telle, mais d'une intervention et d'une transformation permanentes : s'il va de soi que le cadre est variable au fil des saisons, les éléments changent eux aussi, certains apparaissent, d'autres disparaissent et leur disposition est régulièrement modifiée. Le Toutou, qui évolue dans un paysage naturel et contribue à sa métamorphose, pourrait-il se rattacher d'une certaine façon à certaines formes d'art parmi les plus actuelles: installations mobiles, expériences sensorielles, œuvres modulables, créations participatives? Au moins peut-on l'associer à l'art interactif, qui définit l'œuvre comme un processus au cours duquel le créateur travaille à la construction du sens. De plus, par sa dimension sociale, on peut assurément l'associer à un art interactif collectif.

\section{Le Toutou est-il une œuvre d'art?}

Cette question, nous le savons, fait partie de celles que l'on évite de poser lors d'un repas de famille, puisqu'en l'absence de définition consensuelle de l'art, tout accord est impossible. Toutefois, si l'on tient compte du fait qu'une des spécificités de l'art contemporain est le refus de toute limite à la création, et que l'œuvre d'art est une création qui vise autant l'intellect que la sensibilité, alors rien ne permet de lui dénier cette qualité. Ce qui est patent, c'est que Le Toutou n'a rien d'un dépôt d'ordures ni 
d'une opération de ramassage de doudous, semblable à celles qui s'organisent çà et là (par exemple, dans la ville de Québec à l'occasion de la Parade des jouets annuelle).

Nous avons relevé ci-dessus plusieurs traits qui le rapprochent de l'art contemporain et il est certain qu'il ne manquera pas d'évoquer pour les amateurs diverses notions ou divers courants artistiques tels que : art brut, ready-made, pop'art, land art, œuvre in situ, art environnemental, art éphémère, récup-art, etc. De même, il ravira tous ceux qui voient avant tout dans l'art un questionnement : sur nous-mêmes, sur le monde, et sur son propre statut.

21 Aurions-nous donc affaire à un objet artistique non identifié, un OANI (acronyme réorthographié WANI par Paul Ardenne et Marie Maertens à l'occasion de l'exposition organisée sur ce thème à la Fondation Ricard à Paris en 2011) ? En fait, même si Le Toutou n'a, en première analyse, rien à voir avec la dérive, forme de flânerie existentielle prônée par Guy Debord (1956) - rien d'urbain notamment -, c'est bien à une œuvre situationniste qu'il nous fait plutôt songer, par cette particularité qu'il a d'offrir à un sujet collectif constitué par le hasard un objet purement opportuniste et d'activer ainsi une potentialité insoupçonnée du territoire public. En effet, au bord de la route 117 , Le Toutou crée un espace commun à des individus indéterminés qui constituent « une communauté [d'action] en devenir, dont les contours se redéfinissent à chaque occasion [...] et dont les membres ne sont en réalité liés que par le seul partage temporaire [d'une action] » (Lambert, 2015). Encore faudrait-il pourtant qu'il y ait un auteur, une intention revendiquée, ce qui n'est pas le cas ici.

Nous en sommes donc réduits à prendre Le Toutou tout simplement comme il s'offre à nous, c'est-à-dire comme une création collective, proliférante et évolutive, sans objectif exprimé, authentique et spontanée.

\section{Et l'environnement?}

23 À première vue, Le Toutou rappelle l'œuvre d'Iain Baxter, artiste canadien contemporain, qui présentait en 1999 une installation constituée de peluches-animaux. Disposées sur de nombreuses étagères, elles étaient enfermées dans des bocaux à conserves emplis d'eau distillée. Devant ce rassemblement de peluches « emprisonnées ", il n'était pas difficile de comprendre que l'artiste se questionnait, et nous questionnait, sur la relation animaux-conservation-conserves, l'œuvre faisant référence aux problèmes environnementaux qui le préoccupent.

Même si Le Toutou n'a pas d'intention militante explicite, c'est une œuvre qui transforme elle aussi nos idées en art : un art qui crée de la magie, qui fait appel à nos souvenirs, qui interroge nos attitudes, un art qui œuvre dans et - nous le croyons pour l'environnement. Le Toutou rejoint également une autre idée de Baxter qui invitait les voyageurs à commencer à regarder un segment de paysage, en sollicitant nos émotions, l'imaginaire et la réflexion.

Ce que l'on peut tirer de cette rencontre, c'est que Le Toutou offre la possibilité de faire valoir la nature fondamentalement sensible de l'humain. L'œuvre montre également que les citoyens sont prêts à s'engager dans des pratiques participatives, en format " libre choix ", et ceci spontanément, au-delà des actions institutionnalisées, planifiées et concertées. En ce sens, elle rappelle la démarche de l'ASC (art pour le changement social), mouvement qui prône une participation de tout un chacun à la création dans le 
but de favoriser un changement social qui vise, entre autres, l'évolution des politiques environnementales ${ }^{6}$. Dans un contexte où les partisans de la protection de la nature dénoncent constamment les dissonances existant entre un désir sociétal d'adopter des actions plus favorables à l'environnement et le manque d'engagement à cet effet, l'apparition du Toutou est particulièrement intéressante.

Cette analyse nous oriente vers deux perspectives à portée éducative qui peuvent être associées au phénomène Toutou.

La première est liée au public qui gravite autour de l'œuvre, créateurs et spectateurs confondus. Pour eux, vivre une telle expérience sociale revient à aviver une mémoire sensible (personnelle ou collective). Le Toutou dépasse ici la dimension ludique, esthétique ou contemplative pour engager le questionnement de la personne sur son rapport à l'environnement: le paysage devient le théâtre d'une mise en scène qui stimule l'imaginaire du rapport à la nature et invite à la réflexion, à la connexion. Cette forme de pratique participative permet, par ses prolongements, un changement relationnel qualitatif entre la créativité, l'individu et la nature.

Une deuxième perspective dans laquelle s'inscrit Le Toutou, et qui nous intéresse particulièrement, concerne les retombées positives possibles dans le milieu scolaire: un projet de médiation/appréciation/création pourrait se construire autour de l'œuvre, offrant plusieurs pistes d'éducation relative à l'environnement. Ce travail ouvrirait, par contact sensible avec l'œuvre et le lieu, des questionnements sur le potentiel d'une telle œuvre - et celui d'autres pratiques artistiques - pour célébrer et soutenir la protection des milieux naturels. Approfondir, à travers le regard, la réflexion sur le rapport de l'humain à la Terre, amener les participants à s'interroger sur leurs propres attitudes, à développer de l'empathie, c'est aborder le vaste domaine des valeurs environnementales, au fondement des comportements individuels et collectifs à l'égard de la nature. Cette approche rejoint le concept du changement émergent qui relève d'un paradigme [...] fondamentalement positif, centré sur l'émergence, donc sur ce que l'énergie, la volonté, l'émotion et la créativité d'un groupe ou d'une communauté peuvent accomplir pour favoriser une intelligence collective éclairée. [On y explore] pourquoi et comment une personne ou une communauté s'engagent avec cœur, de manière responsable et durable à changer le monde en changeant d'abord elle-même (Mahy et Carle, 2012, p. 4).

Enfin, il s'agit d'exploiter ce lien particulier qui se crée entre l'individu, l'œuvre et le milieu, lien qui s'avère à la fois affectif, imaginaire et social, et qui favorise la mise en place de ce qu'Alain Kerlan définit comme un modèle esthétique d'éducation, ceci dans une optique visant implicitement l'ERE.

\section{Pour conclure : Le Toutou a-t-il un avenir ?}

Aujourd'hui, Le Toutou a toutes les apparences d'un petit écosystème vivant. Il accueille de nouveaux membres, connaît les déboires de la proximité des humains (vol d'objets par exemple) et subit durement les intempéries de la nature : la pluie, le vent, les rigueurs de l'hiver. Même si un antique écran cathodique d'ordinateur est posé devant elles, les peluches du Toutou nous font face, elles nous regardent (libre à chacun d'imaginer ce qu'elles voient) ; elles dialoguent avec nous, fugitivement, tout en nous renvoyant l'image d'une société conviviale, chaleureuse, qui éveille, dans une 
apparence d'abondance et de sérénité, une nostalgie d'enfance et de famille. C'est pourquoi Le Toutou de La Vérendrye attire naturellement la sympathie.

Pour l'heure, il ne connait toutefois qu'une forme d'évolution: l'accroissement par prolifération et il est peu probable qu'il expérimente un développement différent en l'absence d'intervention volontariste. On peut donc penser que tôt ou tard ses ambitions expansionnistes se heurteront au propriétaire ou au gardien des lieux, avec comme perspective la destruction ou l'institutionnalisation.

L'avenir le dira. Jusque-là, réjouissons-nous de le voir apporter pour le moins un peu de fantaisie dans un lieu où règne l'uniformité du paysage routier.

\section{BIBLIOGRAPHIE}

Bertini, M.-J. (2015). Figures de l'anonymat. De quoi Banksy est-il le non ? Cahiers de Narratologie, (29). Récupéré le 18 avril 2018 de : http://journals.openedition.org/narratologie/7398

Clément, G. (s. d.). Le Tiers-Paysage. Récupéré le 8 décembre 2017 de : http:// www.gillesclement.com/cat-tierspaysage-tit-le-Tiers-Paysage

Clément, G. (2016). Manifeste du Tiers paysage. Saint-Germain-sur-Ille : Éditions du commun. Récupéré le 8 août 2018 de : http://www.editionsducommun.org/download/335.

Debord, G. (1956). Théorie de la dérive. Les Lèvres nues, n 9, nov. 56, 6-10.

République française, ministère des Transports et ministère de la Culture et de la Communication. (1980). « Travaux de décoration au titre du 1 p 100 dans les constructions réalisées par le ministère des transports ». Arrêté du 18 juin. Journal officiel de la République française, 16 juillet 1980, p. 56296-56297. Récupéré le 7 août 2018 de : https:// www.legifrance.gouv.fr/jo_pdf.do ?id =JORFTEXT000000491901\&pageCourante =56296

Lambert, B. (2015). Qu'est-ce qu'une communauté narrative ? Communication présentée au colloque du Centre de recherche sur le texte et l'imaginaire (Figura) : Les imaginaires de la communauté, le 4 mars 2015, Montréal. Récupéré le 11 novembre 2017 de l'observatoire de l'imaginaire contemporain : http://oic.uqam.ca/fr/communications/quest-ce-quune-communaute-narrative Kerlan, A. (2004). L'art pour éduquer ? La tentation esthétique. Québec : Presses de l'Université Laval. Mahy, I. et Carle P. (2012). Théorie U, changement émergent et innovation. Québec : Presses de l'Université du Québec.

\section{NOTES}

1. http://ville.montreal.qc.ca/375/legs/bleu-de-bleu / https://www.bnc.ca/a-propos-de-nous/ bleu-de-bleu.html.html

2. https://truck-stop.ca/cest-quoi/

3. Le mot toutou - doudou en français hexagonal - désigne la peluche fétiche à laquelle s'attachent souvent les jeunes enfants. 
4. Les réserves fauniques du Québec ont été créées en vertu de la Loi sur la conservation et la mise en valeur de la faune et relèvent de la responsabilité du ministre des Forêts, de la Faune et des Parcs (MFFP). Ce sont des terres provinciales dont la gestion est assurée par la Société des établissements de plein air du Québec (Sépaq, https://www.sepaq.com). Elles sont «vouées à la conservation, à la mise en valeur et à l'utilisation de la faune ainsi qu'accessoirement à la pratique d'activités récréatives ». Les activités forestières et minières n'y sont pas interdites, mais elles sont rigoureusement contrôlées.

5. Toutes les photos sont de l'auteure.

6. Sur l'ASC, voir https://icasc.ca/sites/default/files/resource_attachments/ stateoftheart_fr_0.pdf

\section{RÉSUMÉS}

L'art routier est sans doute né avec les voies de communication, mais il a connu récemment de nombreux développements. Après en avoir donné quelques exemples, nous nous intéresserons plus particulièrement à une création qui est née au cœur de la réserve faunique La Vérendrye au Québec sur le bord de la route 117. Il s'agit d'un " rassemblement » apparemment spontané de peluches au milieu du paysage, sans auteur ni objectif défini. Nous nous interrogerons dans un premier temps sur les affinités de cette installation avec diverses formes d'art et examinerons ensuite le lien qu'elle peut avoir avec l'éducation relative à l'environnement avant de nous poser la question de son devenir.

Roadside art may have originated with the development of road networks, but it has recently undergone a constant increasing. After giving some examples, we will focus on a creation that was born in the heart of the La Vérendrye Wildlife Reserve, Quebec, on the edge of Highway 117. This is an apparently spontaneous "gathering" of soft toys in the middle of the landscape, without author or definite purpose. We will point out its similarities with contemporary art, and ask ourselves if it can be considered as an art work, and of what type. We will then examine the link it may have with environmental education, before questioning its future.

\section{INDEX}

Index géographique : Québec

Keywords : installation art, environmental education, roadside art, third landscape

Mots-clés : art routier, environnement, installation, tiers-paysage, éducation relative à

l'environnement

\section{AUTEUR}

\section{MAIA MOREL}

Maia Morel est professeure régulière en didactique des arts plastiques, Université du Québec en Abitibi-Témiscaminque. Ses intérêts de recherche couvrent la didactique des arts visuels, l'art et 
l'éducation au vivre-ensemble, l'enseignement artistique et culturel dans une perspective internationale. 